\title{
Reducing Missed Opportunities for Influenza Vaccination in Patients with Rheumatoid Arthritis: Evaluation of a Multisystem Intervention
}

\author{
Rachel Broderick, Iazsmin Ventura, Sunoz Soroosh, Lourdes Franco, and Jon T. Giles
}

ABSTRACT. Objective. To assess a multimodal intervention for reducing missed opportunities for outpatient influenza vaccination in individuals with rheumatoid arthritis (RA).

Methods. Patients with RA were enrolled from a single center and each rheumatology outpatient visit was tracked for missed opportunities for influenza vaccination, defined as a visit in which an unvaccinated patient without contraindications remained unvaccinated or lacked documentation of vaccine recommendation in the electronic medical record (EMR). Providers then received a multimodal intervention consisting of an education session, EMR alerts, and weekly provider-specific e-mail reminders. Missed opportunities before and after the intervention were compared, and the determinants of missed opportunities were analyzed.

Results. A total of 228 patients with RA were enrolled (904 preintervention visits) and 197 returned for at least 1 postintervention visit (721 postintervention visits). The preintervention frequency of any missed opportunities for influenza vaccination was $47 \%$. This was reduced to $23 \%$ postintervention $(\mathrm{p}<0.001)$. Among those vaccinated, the relative hazard for influenza vaccination post- versus preintervention period was $1.24(\mathrm{p}=0.038)$. Younger age, less frequent office visits, higher erythrocyte sedimentation rate, and negative attitudes about vaccines were each independently associated with missed opportunities preintervention. Postintervention, these factors were no longer associated with missed opportunities; however, the intervention was not as effective in non-Hispanic black patients, non-English speakers, those residing outside of the New York City metropolitan area, and those reporting prior adverse reactions to vaccines.

Conclusion. Improved uptake of influenza vaccination in patients with RA is possible using a multimodal approach. Certain subgroups may need a more potent intervention for equivalent efficacy. (First Release May 15 2018; J Rheumatol 2018;45:1220-8; doi:10.3899/jrheum.170763)

\section{Key Indexing Terms: IMMUNIZATION PREVENTIVE HEALTH \\ RHEUMATOID ARTHRITIS ELECTRONIC MEDICAL RECORD}

\section{CLINICAL PRACTICE DELIVERY OF CARE}

Individuals with rheumatoid arthritis (RA) are at a higher risk of developing influenza than those without RA, and complications from the infection are more frequent in $\mathrm{RA}^{1}$. Although individuals with RA have been shown to mount protective responses after influenza vaccination, even among

From the Division of Rheumatology, Columbia University, College of Physicians and Surgeons, New York, New York, USA.

Supported by an unrestricted research grant from Pfizer as part of the Pfizer Independent Grants for Learning and Change program.

$R$. Broderick, RS, Division of Rheumatology, Columbia University, College of Physicians and Surgeons; I. Ventura, MD, Division of Rheumatology, Columbia University, College of Physicians and Surgeons; S. Soroosh, RN, Division of Rheumatology, Columbia University, College of Physicians and Surgeons; L. Franco, BA, Division of Rheumatology, Columbia University, College of Physicians and Surgeons; J.T. Giles, MD, MPH, Division of Rheumatology, Columbia University, College of Physicians and Surgeons.

$R$. Broderick and I. Ventura contributed equally to the manuscript and should be considered co-primary authors.

Address correspondence to Dr. J.T. Giles, Division of Rheumatology, Columbia University, College of Physicians and Surgeons, 630 W. 168th St., Physicians and Surgeons Building, Suite 10-445, New York, New York 10032,USA.E-mail: jtg2122@columbia.edu

Accepted for publication February 12, 2018. those receiving biologic immunomodulators ${ }^{2}$, vaccination rates are suboptimal ${ }^{3,4,5}$ and no better than in the non-RA population, despite a general recognition of higher risk. Current guidelines ${ }^{6}$ recommend yearly influenza vaccination for individuals with RA with no restriction based on age, disease factors, or concomitant medications.

There are many potential barriers to effective vaccine uptake in clinical practice, including patient and provider perceptions of risk, attitudes about the efficacy and safety of vaccines, logistical barriers related to the delivery of care within a practice, and financial barriers. Many of these barriers are known to be more prevalent in racial/ethnic minority groups and those who are economically disadvan$\operatorname{taged}^{7}$. Moreover, priorities for vaccination may be decreased at the point of care in patients with acute issues related to their RA disease management or comorbidities, a group in which vaccination should be a priority because of the increased risk of infection among patients with RA with more active and severe disease ${ }^{8}$. Interventions to increase vaccine uptake require assessment of how these barriers affect vaccine uptake within a given practice.

Personal non-commercial use only. The Journal of Rheumatology Copyright @ 2018 . All rights reserved. 
A number of practice-based interventions aimed at improving vaccination rates in the general population ${ }^{9}$ and in people with $\mathrm{RA}^{10,11}$ have been reported. A prior study ${ }^{11}$, conducted in a rural, community-based rheumatic disease population with a highly integrated healthcare delivery system, reported a significant increase in RA vaccination rates using alerts integrated into the electronic medical record (EMR) system, although more than one-third of the patients in the study remained unvaccinated. Modest improvements using EMR alerts have also been shown in other diseases ${ }^{12}$, with the most effective interventions involving multiple modalities in addition to EMR alerts. In general, modalities that integrate nonphysician personnel into vaccine delivery and/or involve active participation of patients are more effective than those that involve only alerts to the provider at the point of care $^{9}$. While such a multimodality was effective in RA for uptake of some vaccines ${ }^{10}$, there was no clear-cut evidence of efficacy in optimizing influenza vaccination in a recent study.

Most studies of interventions to increase vaccine uptake have analyzed overall vaccine uptake rates as the primary measure of the efficacy. However, influenza is a seasonal disease with incidence peaking in the winter months. Vaccine becomes available in the late summer and immunity does not develop until several weeks after receipt of the vaccine. Thus, delay in vaccination can lead to risk, and vaccine received late during influenza season is suboptimal ${ }^{13}$. For this reason, any missed opportunities at the point of care for administering or recommending vaccination may place the patients at higher risk, and optimizing care includes both increasing overall vaccination rates and ensuring that vaccination occurs as early during the influenza season as possible.

For our study, we compared the frequency of missed opportunities for influenza vaccination before and after a multimodal intervention involving provider education, EMR-based alerts, and weekly provider-specific e-mail reminders in a large, urban, academic, tertiary healthcare facility with a large proportion of minority and economically disadvantaged patients. We hypothesized that the intervention would result in a decrease in missed opportunities for influenza vaccination, but that differences in the efficacy of the intervention would be observed based on sociodemographic strata.

\section{MATERIALS AND METHODS}

Study participants. Patients with RA followed as outpatients at Columbia University Medical Center (CUMC) were recruited during their routinely scheduled outpatient appointments beginning after September 1, 2013. To construct an unbiased and generalizable sample, there were few exclusions to enrollment. Patients were included if they had a diagnosis of RA (defined as having a diagnostic code for RA in the EMR that was assigned by the treating CUMC rheumatologist), were 18 years of age or older, and received primary outpatient rheumatology specialty care for RA at either the CUMC Faculty Practice or the Fellow's Clinic. Patients were excluded if they had a contraindication to receiving influenza vaccine (i.e., allergy or intolerance), planned to discontinue care at CUMC, or planned to move out of the area during the study interval. All patients provided written informed consent prior to undergoing any study procedures, and the study was approved by the CUMC Institutional Review Board (Protocol Number AAAM2252). Because the rheumatology providers who participated in study-specific education sessions were the primary target of the intervention, and had study-associated data collected about them, written informed consent was also obtained from all of the practicing CUMC rheumatology faculty and fellows.

Study timing and intervention. The study occurred in 2 phases lasting 12 months before and after the intervention. The preintervention phase began with an educational session for the providers focused on the rationale, efficacy, and recommended uses of vaccination in patients with RA. The goal of the education session, which occurred just prior to September 1, 2013, was to normalize baseline knowledge across the providers. The education session was based on the most recent Advisory Committee on Immunization Practices and American College of Rheumatology guidelines ${ }^{6,14}$. No other reminders or prompts about vaccination were provided over the ensuing 12 months in which patients with RA were enrolled into the study. In August 2014, enrollment concluded and all of the enrolled patients with RA were contacted by phone and queried on the type (intradermal vs intranasal) and date they had received vaccines in the prior 12 months. The patients with RA cared for at CUMC obtained influenza vaccine from a variety of sources, which can include providers at our medical center or at other facilities. An increasingly common location for obtaining vaccinations is pharmacies. Less common are vaccine drives at events, community centers, or through the Public Health Department. While some of these efforts distribute the vaccine free of charge, most are reimbursed through public or private insurance. When contacted, we queried patients about the setting in which they received the vaccine. Using these data, EMR records for each of the patient's outpatient visits over the prior 12 months were examined for missed vaccination opportunities. An outpatient visit was designated as a missed opportunity if an unvaccinated patient eligible for vaccination did not have a documented influenza vaccination administered on the day of the visit or the visit note did not document that influenza vaccination was recommended. Patients eligible for influenza vaccination were those who had not received influenza vaccination already or had no documented contraindications to vaccination. Visits in which patient refusal of vaccination was documented were not classified as missed opportunities. Missed opportunities for influenza vaccination were considered only between September 1 and April 30.

The intervention began on September 1, 2014. Just after this date, the providers underwent another educational session with the same educational content as the preintervention session, along with an introduction to the EMR-based alerts and study notifications that were to occur. They were also instructed on how to document in the EMR where and when a vaccination outside CUMC occurred. Thereafter, an alert was entered into the EMR for every enrolled patient reading, "REMINDER: INQUIRE, ORDER, and RECORD IMMUNIZATIONS." The alert was prominently displayed in red lettering on the main patient-specific EMR navigation screen. In addition, study personnel tracked the scheduling and documented immunization status for enrollees in the EMR, and each provider was notified weekly by e-mail of which enrolled patients with RA were scheduled to have an outpatient rheumatology visit with them for the coming week, their immunization status, and personalized encouragement based on their performance recommending, administering, and documenting vaccines. Alerts and e-mail reminders continued through the beginning of May 2015. Enrollees were again contacted in August 2015 and their vaccine experience from the prior 12 months was documented, from which missed opportunities for influenza vaccination were calculated for the postintervention period.

Data collection. At baseline, enrolled patients were administered questionnaires on demographics, education, primary language spoken, and minutes of morning stiffness. The Health Assessment Questionnaire ${ }^{15}$ was completed and used to estimate physical disability. General health and pain level over the previous week were assessed using the $100-\mathrm{mm}$ visual analog scale. A Vaccine Attitudes Questionnaire ${ }^{16}$ was administered to assess general

Personal non-commercial use only. The Journal of Rheumatology Copyright (c) 2018. All rights reserved. 
attitudes about the safety and efficacy of vaccines. Prior vaccinations were recorded, as was whether the patient had ever had a serious infection (hospitalized or nonhospitalized) or an adverse reaction to a vaccine. Baseline data were collected from the EMR on the number of comorbid conditions, insurance coverage, RA disease characteristics (RA duration, documentation of active synovitis, use of biologic and nonbiologic disease-modifying antirheumatic drugs, prednisone, and nonsteroidal antiinflammatory drugs), and frequency of outpatient rheumatology visits. The most recent standard-of-care laboratory assessments and inflammatory markers were recorded from the EMR.

Statistical analysis. The crude influenza vaccination rate and the frequency of pre- and postintervention missed opportunities were determined and their associated $95 \%$ CI calculated. Among those vaccinated, time to vaccination was modeled using Cox proportional hazards modeling, with the hazard of influenza vaccination pre- and postintervention compared. The independent associations of participant characteristics with the frequency of missed vaccination opportunities before and after the intervention were modeled using ordinary logistic regression first in crude (unadjusted) models. Characteristics associated with the outcome at the $\mathrm{p}<0.20$ level, to allow for residual confounding, were carried into multivariable models. Noncontributory covariates were excluded using the likelihood ratio test for nested models and the predictability of the model was tested by calculating the area under the curve (AUC) of the receiver-operator function. All statistical calculations were performed using Intercooled Stata 12 (StataCorp). A 2-tailed $\alpha$ of 0.05 was used throughout.

\section{RESULTS}

Baseline characteristics of the 228 enrolled patients are summarized in Table 1. A broad range of sociodemographics and RA characteristics was represented. There were 904 preintervention visits, with a median of 4 preintervention visits per patient. A total of 197 patients with RA (86\%) had at least 1 visit after the intervention occurred, contributing 721 postintervention visits, with a median of 3 postintervention visits per patient. The baseline characteristics of the subset that returned postintervention did not differ substantially from those of the originally enrolled group (Table 1).

Efficacy of the intervention. The preintervention frequency of any missed opportunities for influenza vaccination was $47 \%$. This was reduced to $23 \%$ after the intervention (Figure $1 \mathrm{~A})$, resulting in an average of 0.59 fewer missed opportunities ( $\mathrm{p}<0.001$; data not shown). Among those with any preintervention missed opportunities for influenza vaccination who returned after the intervention $(n=93), 59(63 \%)$ had no missed opportunities after the intervention. In contrast, only 12 of the 104 with no missed opportunities for influenza vaccination in the preintervention period had a missed opportunity after the intervention $(12 \%$; $\mathrm{p}<0.001$; data not shown). Among those vaccinated, the crude relative hazard for influenza vaccination comparing time to vaccination for the post- versus preintervention period was 1.24 $(\mathrm{p}=0.038)$, indicating significantly less time to vaccination associated with the intervention (Figure 1B).

Predictors of missed influenza vaccination opportunities in the preintervention period. After modeling the baseline characteristics detailed in Table 1 , there were 4 characteristics significantly and independently associated with missed opportunities for influenza vaccination in the preintervention period (Table 2): younger age, less frequent office visits, higher erythrocyte sedimentation rate (ESR), and more negative attitudes about vaccines. No other demographic, practice, or RA disease characteristics were associated with missed opportunities. For the 4 identified factors, the AUC for predicting who would have any preintervention missed opportunities for influenza vaccination was 0.724 (95\% CI $0.649-0.800)$. None of these factors remained significantly associated with missed opportunities for influenza vaccination after the intervention.

The change in the frequency of missed opportunities for influenza vaccination before and after the intervention according to these factors is shown in Figure 2. Prior to the intervention, 40-50\% of the younger patients with RA (age $<60 \mathrm{yrs}$ ) had missed opportunities for influenza vaccination which were markedly reduced after the intervention (Figure 1A). Missed opportunities were also reduced after the intervention for the group age 60-69 years. For the group age 70 years and above, the preintervention rate of missed opportunities was the lowest compared with the younger age groups (22\%); however, the rate of missed opportunities numerically increased for this group after the intervention (39\%), but was not significantly different from the preintervention rate. Prior to the intervention, patients with less frequent visits had a higher rate of missed influenza vaccination opportunities compared with those with visits occurring more frequently than every 3 months (Figure 2B). After the intervention, the rate was lower for this group, but this difference was not quite statistically significant $(\mathrm{p}=0.081)$. More positive attitudes about general vaccine efficacy and safety were strongly associated with fewer missed influenza vaccination opportunities in the preintervention period (Figure 2C). This trend was eliminated after the intervention, in which even those with less positive attitudes had nearly the same rate of missed opportunities as those with more positive attitudes. Finally, having an elevated ESR was associated with missed influenza vaccination opportunities before the intervention (Figure 2D); however, after the intervention, those with higher ESR levels had nearly the same rate of missed opportunities as those with lower levels ( $15 \%$ vs $23 \%$, respectively; $\mathrm{p}=0.58)$.

Predictors of missed influenza vaccination opportunities in the postintervention period. Despite improvement in influenza vaccination uptake for the total group, there were subgroups of patients in which the intervention was not as efficacious in reducing missed vaccination opportunities (Modeled in Table 2 and depicted graphically in Figure 3). Preintervention, there was no significant difference in the frequency of missed opportunities for influenza vaccination according to race/ethnicity, although numerically the highest frequency of missed opportunities was observed in the non-Hispanic white group (Figure 3A). After the intervention, there was a significant decrease in the frequency of missed opportunities in the non-Hispanic white and Hispanic groups, but no decrease in the non-Hispanic black group.

Personal non-commercial use only. The Journal of Rheumatology Copyright $\odot$ 2018. All rights reserved 
Table 1. Baseline characteristics at enrollment for the total cohort and those returning after the intervention. Values are mean \pm SD unless otherwise specified.

\begin{tabular}{|c|c|c|}
\hline Characteristics & Total, $\mathrm{n}=228$ & Returned after Intervention, $\mathrm{n}=197$ \\
\hline Age, yrs & $58 \pm 15$ & $59 \pm 14$ \\
\hline Female, n (\%) & $199(88)$ & $170(87)$ \\
\hline \multicolumn{3}{|l|}{ Race/ethnicity, n (\%) } \\
\hline NH white & $69(30)$ & $56(28)$ \\
\hline NH black & $36(16)$ & $33(17)$ \\
\hline Hispanic & $104(45)$ & $90(46)$ \\
\hline Other/not declared & $19(9)$ & $18(9)$ \\
\hline Faculty practice, n (\%) & $169(74)$ & $142(72)$ \\
\hline \multicolumn{3}{|l|}{ Primary insurance, $\mathrm{n}(\%)$} \\
\hline Private & $108(47)$ & $91(46)$ \\
\hline Medicare & $77(34)$ & $71(36)$ \\
\hline Medicaid & $44(19)$ & $35(18)$ \\
\hline \multicolumn{3}{|l|}{ Residence, n (\%) } \\
\hline Manhattan & $110(49)$ & $95(49)$ \\
\hline Other borough & $56(25)$ & $51(26)$ \\
\hline Contiguous county & $33(14)$ & $27(14)$ \\
\hline Outside NYC metro & $29(12)$ & $22(11)$ \\
\hline Primary language English, n (\%) & $137(60)$ & $116(59)$ \\
\hline Any college, $\mathrm{n}(\%)$ & $130(57)$ & $110(56)$ \\
\hline Body mass index, $\mathrm{kg} / \mathrm{m}^{2}$ & $28.2 \pm 6.8$ & $28.1 \pm 6.8$ \\
\hline Rheumatology visit at least every 3 mos, $\mathrm{n}(\%)$ & $168(74)$ & $141(72)$ \\
\hline No. problems on problem list, median (IQR) & $6(3-9)$ & $6(4-9)$ \\
\hline RA duration, yrs, median (IQR) & $9.5(4.0-20.0)$ & $10(4-20)$ \\
\hline AM stiffness, min, median (IQR) & $15(5-40)$ & $15(5-30)$ \\
\hline Patient global health, VAS $1-100$, median (IQR) & $75(50-90)$ & $75(50-90)$ \\
\hline Pain, VAS 1-100; median (IQR) & $30(10-60)$ & $30(10-60)$ \\
\hline HAQ, 0-3, median (IQR) & $0.77(0.38-1.31)$ & $0.77(0.31-1.31)$ \\
\hline Synovitis recorded in note, $\mathrm{n}(\%)$ & $121(53)$ & $101(52)$ \\
\hline Any DMARD, n (\%) & $203(89)$ & $175(89)$ \\
\hline Nonbiologics, $\mathrm{n}(\%)$ & $149(65)$ & $130(66)$ \\
\hline Biologics, $\mathrm{n}(\%)$ & $93(41)$ & $80(41)$ \\
\hline Prednisone, $\mathrm{n}(\%)$ & $80(35)$ & $71(36)$ \\
\hline NSAID, n (\%) & $73(32)$ & $57(29)$ \\
\hline ESR, mm/h, median (IQR) & $17(8-32)$ & $18(8-35)$ \\
\hline CRP, mg/l, median (IQR) & $3.4(0.9-8.8)$ & $3.6(1.0-9.2)$ \\
\hline Elevated liver function tests, $\mathrm{n}(\%)$ & $15(7)$ & $15(8)$ \\
\hline White blood cell count, $\times 1000$ & $7.1 \pm 2.3$ & $7.1 \pm 2.4$ \\
\hline Hemoglobin, $\mathrm{mg} / \mathrm{dl}$ & $12.5 \pm 1.4$ & $12.5 \pm 1.5$ \\
\hline Hemoglobin $<10.0 \mathrm{mg} / \mathrm{dl}, \mathrm{n}(\%)$ & $11(5)$ & $11(6)$ \\
\hline Creatinine, mg/dl, median (IQR) & $0.78(0.67-0.90)$ & $0.79(0.66-0.91)$ \\
\hline Creatinine $>1.0 \mathrm{mg} / \mathrm{dl}$ & $39(17)$ & $37(19)$ \\
\hline Prior hospitalized infection, $\mathrm{n}(\%)$ & $48(21)$ & $47(24)$ \\
\hline Prior non-hospital infection, n (\%) & $98(43)$ & $91(46)$ \\
\hline Prior vaccine adverse reaction, $\mathrm{n}(\%)$ & $14(6)$ & $14(7)$ \\
\hline Vaccine Attitude Score, $0-100$ & $50 \pm 9$ & $51 \pm 9$ \\
\hline
\end{tabular}

NH: non-Hispanic; NYC: New York City; IQR: interquartile range; RA: rheumatoid arthritis; VAS: visual analog scale; HAQ: Health Assessment Questionnaire; DMARD: disease-modifying antirheumatic drug; NSAID: nonsteroidal antiinflammatory drug; ESR: erythrocyte sedimentation rate; CRP: C-reactive protein.

There was a drop in the rate in the other/non-declared race/ethnicity group, but it was not statistically significant owing to the small size of this group.

Preintervention, there was no difference in the frequency of missed opportunities for influenza vaccination between the 2 practice settings (Figure 3B). After the intervention, there was a significantly greater drop in missed opportunities among the patients receiving care in the Faculty Practice compared with those cared for in the Fellow's Clinic. Perhaps not surprising, patients reporting adverse reactions to vaccines in the past were more likely to have missed opportunities for influenza vaccination (Figure 3C), and the postintervention difference in missed opportunities between this group and those without prior vaccine reactions was more pronounced and statistically significant. Patients with RA residing outside the New York City metropolitan area did not have a reduction in missed opportunities for influenza vaccination, compared with significant decreases among those living in Manhattan or other boroughs of New York City (Figure 3D). The reduction in

Personal non-commercial use only. The Journal of Rheumatology Copyright @) 2018. All rights reserved. 


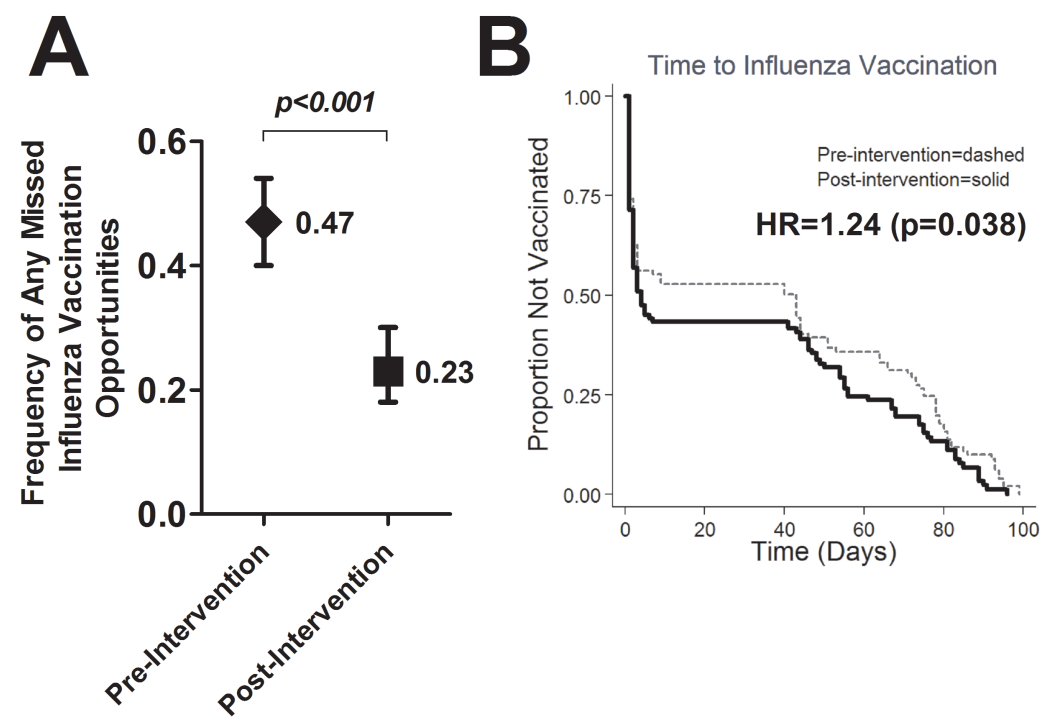

Figure 1. Efficacy of a multisystem intervention to improve influenza vaccination among patients with rheumatoid arthritis. In panel A, the average frequency and $95 \% \mathrm{CI}$ of having any rheumatology outpatient visits deemed as missed opportunities is depicted in the year before and after the intervention. In panel B, the time to vaccination among those eventually vaccinated is depicted, with the HR comparing pre- versus postintervention derived from Cox proportional regressions modeling.

Table 2. Multivariable predictors of having missed opportunities for influenza vaccination before and after the intervention.

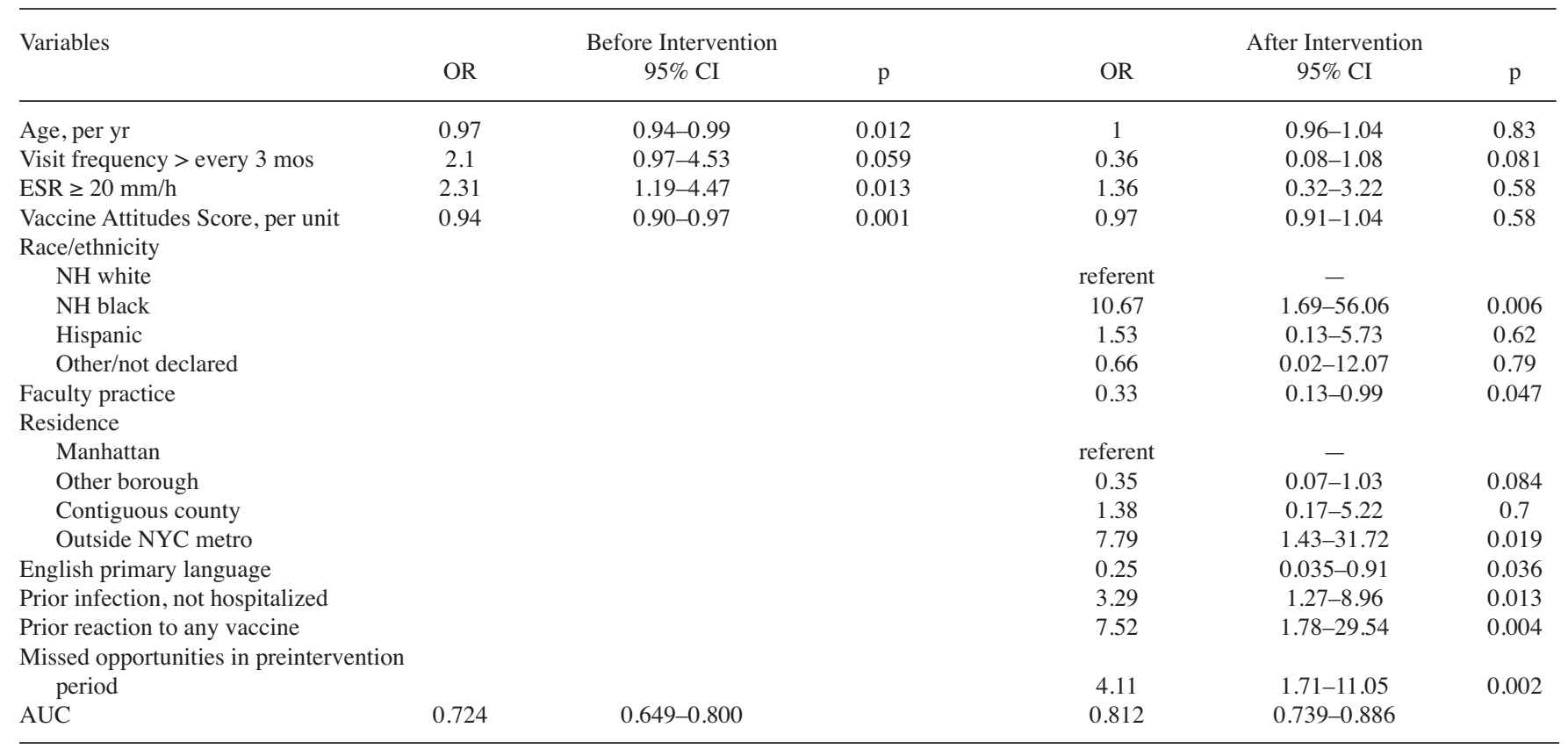

ESR: erythrocyte sedimentation rate; NH: non-Hispanic; NYC: New York City; AUC: area under the receiver-operator curve.

missed opportunities was not as large for patients with RA with a non-English primary language (Figure 3E) and the relative decrease in missed opportunities was not as great among patients reporting versus not reporting a prior nonhospitalized infection (Figure 3F), although the absolute reduction was similar. Even after adjusting for the above characteristics, having any preintervention missed opportunities was one of the strongest predictors of having postintervention missed opportunities (Figure 4), with having just 1 preintervention missed opportunity being as strong a predictor of having any postintervention missed opportunities as having 2 or more preintervention missed opportunities. 

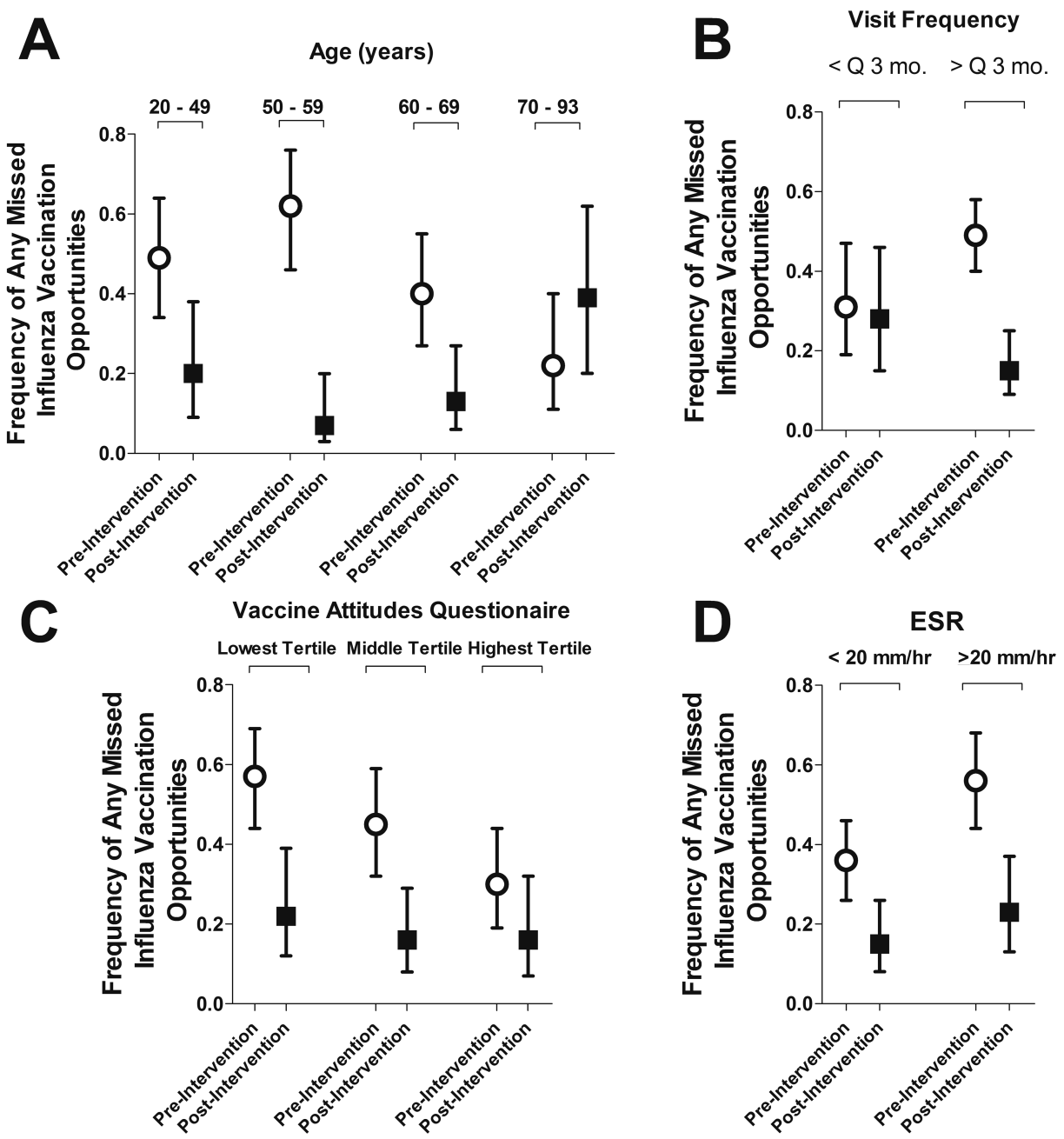

Figure 2. Differences in the frequencies of missed opportunities before and after a multisystem intervention to improve influenza vaccination among patients with rheumatoid arthritis according to characteristics with differential effects preintervention. Pre- and postintervention average frequencies and $95 \%$ $\mathrm{CI}$ are depicted according to age groups (Panel A), visit frequency (Panel B), tertiles of vaccine attitudes (from a vaccine attitudes questionnaire, Panel C), and baseline ESR dichotomized at $20 \mathrm{~mm} / \mathrm{h}$ (Panel D). Graphs are derived from the multivariable logistic regression model detailed in Table 2 (Before Intervention model). ESR: erythrocyte sedimentation rate.

\section{DISCUSSION}

Using a provider-directed intervention involving education, EMR-based alerts, and personalized e-mail reminders, we observed an improvement in the overall rate of influenza vaccination and less delay in the time to vaccination among patients with RA, manifested in a more than $50 \%$ reduction in the frequency of missed point-of-care opportunities for vaccination. Factors associated with missed vaccination opportunities before the intervention (younger age, low visit frequency, elevated ESR, and more negative vaccine attitudes) were abrogated by the intervention. However, the intervention did not benefit all patients with RA equally, because no improvement in missed opportunities was observed among non-Hispanic black patients and those living outside the New York City metropolitan area, and less robust improvements were observed among those cared for in the Fellow's Clinic, those reporting prior adverse reactions to vaccines, and those with primary languages other than English.

Based on provider feedback in our study, the weekly e-mail reminder was the component of the intervention that was the most effective. Although removed from the point of care, the e-mails were personalized, not excessively detailed, and frequent enough to serve as a reminder and general prompt. In contrast, the EMR reminders were deemed less effective because they were easily ignored, despite being prominently displayed on the anchor EMR chart page. The ability to mentally block out EMR reminders has been described as a limit to their efficacy ${ }^{17}$, especially in EMR with many such alerts that may lead to what has been

$$
\text { Personal non-commercial use only. The Journal of Rheumatology Copyright } \odot \text { 2018. All rights reserved. }
$$



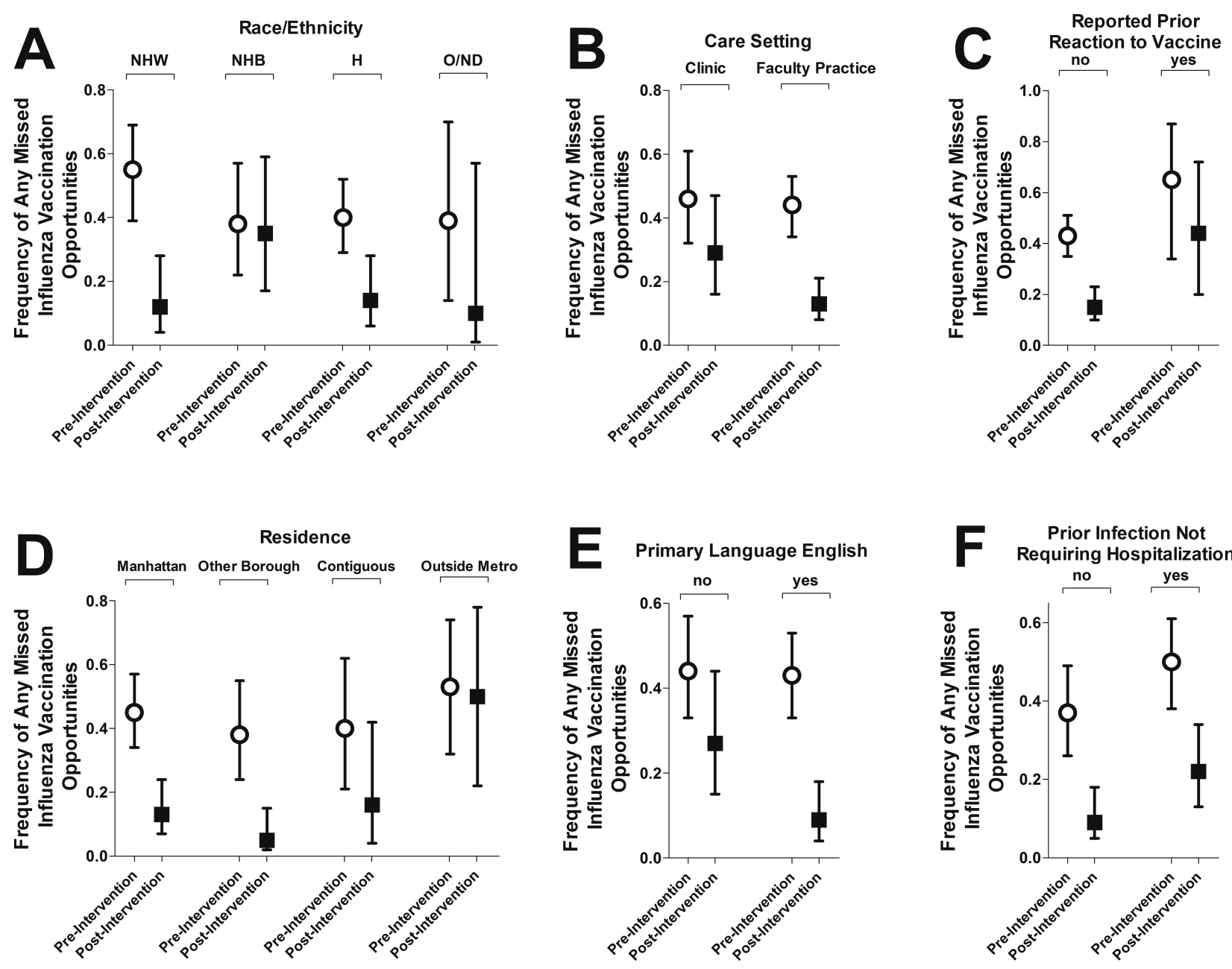

Figure 3. Differences in the frequencies of missed opportunities before and after a multisystem intervention to improve influenza vaccination among patients with rheumatoid arthritis according to characteristics with differential effects postintervention. Pre- and postintervention average frequencies and $95 \%$ CI are depicted according to race/ethnicity (Panel A), care setting (Panel B), self-reported prior nonallergic reaction to any vaccine (Panel C), residence (Panel D), primary language (Panel E), and self-reported prior infection that did not require hospitalization (Panel F). Graphs are derived from the multivariable logistic regression model detailed in Table 2 (After Intervention model). NHW: non-Hispanic white; NHB: non-Hispanic black; H: Hispanic; O/ND: other/not declared.

described as "alert fatigue." Ledwich, et al ${ }^{11}$ reported higher overall vaccination rates among patients with RA in which EMR alerts were directed to physician and nonphysician caregivers (i.e., practice nurses). However, in a more recent study ${ }^{10}$, EMR reminders combined with linked order sets and physician auditing and feedback was not associated with an increase in influenza vaccination, although it should be noted that influenza vaccination was already high in this cohort, and improvements in pneumococcal and zoster vaccination were noted using this intervention.

Ultimately, despite marked improvement in vaccination in our study, 1 in 4 still had missed opportunities for influenza vaccination. This was not equally distributed across patients; in particular, missed opportunities were unchanged among non-Hispanic black patients, the only race/ethnic group that did not have a postintervention rate of missed opportunities around $10 \%$. One explanation for this difference could be generally negative vaccine attitudes, socioeconomic disparities, and lower educational attainment in this group among our population. However, the difference was still evident even after accounting for these characteristics, and the intervention was effective for the Hispanic group, which shares many of these characteristics, on average, with the non-Hispanic Black population cared for at CUMC. Future work could target this group for a different and/or more potent intervention.

Our intervention was successful in abrogating several prominent reasons for missed opportunities, among which younger age, less frequent visits, and higher disease activity have been identified in prior studies ${ }^{3,9,18}$. Notably, the intervention was successful in improving vaccine uptake among patients with less favorable attitudes about the safety and

Personal non-commercial use only. The Journal of Rheumatology Copyright @ 2018. All rights reserved. 


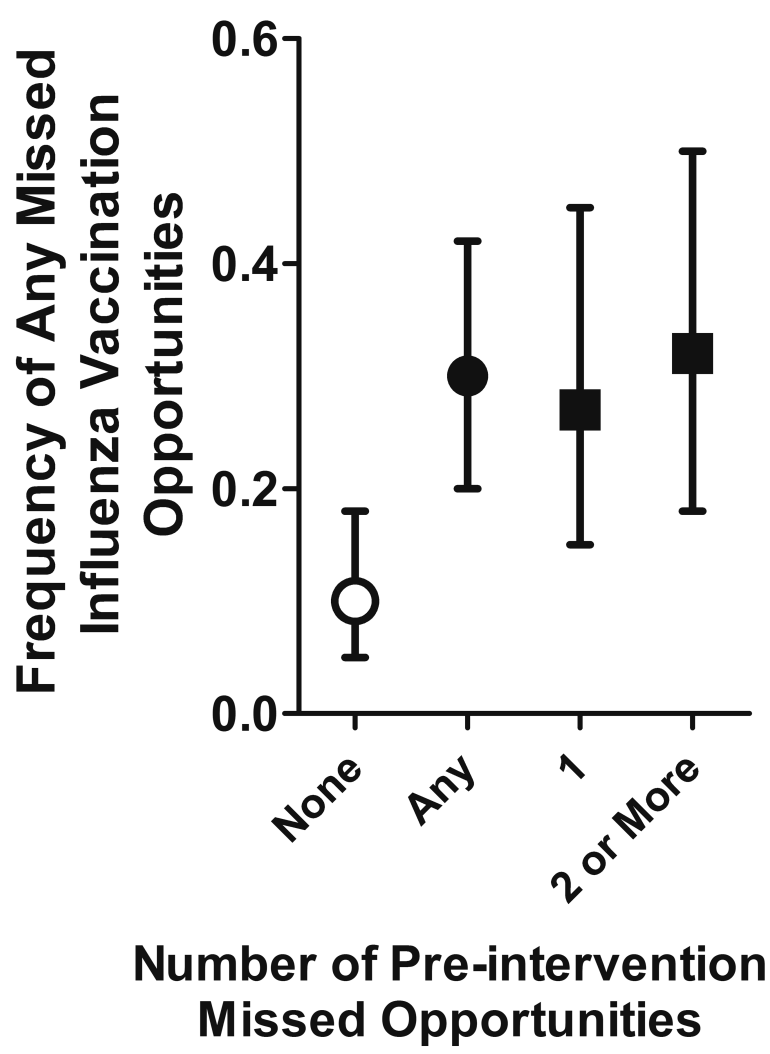

Figure 4. Association of the frequency of missed opportunities for influenza vaccination after a multisystem intervention according to the frequency of missed opportunities before the intervention. Average frequencies and $95 \%$ CI are depicted, adjusted for covariates in the multivariable logistic regression model detailed in Table 2 (After Intervention model).

efficacy of vaccines. Patient perception of vaccine efficacy and safety is complex, and in 1 study $^{19}$ did not appear to be influenced by RA patients' perception of infection risk or treatment with biologics. From our study, the negative effect of vaccine attitudes on a patient's decision to be vaccinated appears to be surmountable when providers are more actively engaged in recommending vaccination, as occurred after the intervention. We are currently analyzing our data to determine whether specific subgroups of patients with negative vaccine attitudes were less susceptible to being vaccinated after the intervention.

There are some notable strengths and limitations of our study. Among the strengths, the study population was ethnically and socioeconomically diverse, allowing for the examination of how these factors influenced the efficacy of the intervention. We also collected a variety of patient-level data not available in the EMR, such as vaccine attitudes and self-report of prior infections/vaccine adverse events, which allowed for a richer investigation of individual determinants of vaccination. Accordingly, we were able to define the independent predictors of a large proportion (i.e., AUC of $0.70-0.80$ ) of the reasons for missed vaccination opportu- nities. An additional strength was the high followup rate, with $86 \%$ of enrolled patients having both pre- and postintervention data. Among limitations, we did not study all the patients with RA followed in our center, because patients were individually consented and enrolled in the study. Thus, it is possible that enrolled patients would not be representative of the larger RA population. In particular, our sample could be enriched with those specifically motivated to be vaccinated because of their enrollment/interest in the study. However, while this could result in higher absolute vaccination rates, it would not affect the change in vaccination rates after the intervention, because patients were enrolled up to a year before the intervention occurred and were not exposed directly to any of the components of the intervention. Another limitation is the potential for misclassification of vaccination status. Ours is not a closed health system, and most patients receive components of their healthcare across multiple health delivery systems in and around New York City. We attempted to limit this by contacting patients directly to determine their vaccination status from the prior year. This introduces the possibility of misclassification because of inaccurate recall; however, for influenza vaccination that is given yearly, we would not expect a large proportion of patients to be inaccurate in their recall of receipt of influenza vaccination for that year. It is possible that the timing of vaccination may have been inaccurate; however, we asked patients to specifically note when vaccine was received, and when needed, followup phone calls were made.

We demonstrated that improvement in both the uptake and timing of influenza vaccination is possible among a diverse population of patients with RA cared for at a large, urban, tertiary-care academic medical center. Moreover, we were able to identify factors associated with inadequate vaccination both before and after the intervention that can be used to further improve rates among a patient population at higher risk for both contracting influenza and developing severe manifestations.

\section{ACKNOWLEDGMENT}

We acknowledge the faculty and fellows of the Columbia University Division of Rheumatology who allowed themselves to be studied.

\section{REFERENCES}

1. Blumentals WA, Arreglado A, Napalkov P, Toovey S. Rheumatoid arthritis and the incidence of influenza and influenza-related complications: a retrospective cohort study. BMC Musculoskelet Disord 2012;13:158.

2. Hua C, Barnetche T, Combe B, Morel J. Effect of methotrexate, anti-tumor necrosis factor $\alpha$, and rituximab on the immune response to influenza and pneumococcal vaccines in patients with rheumatoid arthritis: a systematic review and meta-analysis. Arthritis Care Res 2014;66:1016-26.

3. Hmamouchi I, Winthrop K, Launay O, Dougados M. Low rate of influenza and pneumococcal vaccine coverage in rheumatoid arthritis: data from the international COMORA cohort. Vaccine 2015;33:1446-52.

\section{Personal non-commercial use only. The Journal of Rheumatology Copyright @ $\ 2018$. All rights reserved.}


4. Lanternier F, Henegar C, Mouthon L, Blanche P, Guillevin L, Launay $\mathrm{O}$. Low influenza-vaccination rate among adults receiving immunosuppressive therapy for systemic inflammatory disease. Ann Rheum Dis 2008;67:1047.

5. Krasselt M, Ivanov JP, Baerwald C, Seifert O. Low vaccination rates among patients with rheumatoid arthritis in a German outpatient clinic. Rheumatol Int 2017;37:229-37.

6. Singh JA, Saag KG, Bridges SL Jr, Akl EA, Bannuru RR, Sullivan MC, et al. 2015 American College of Rheumatology Guideline for the Treatment of Rheumatoid Arthritis. Arthritis Rheumatol 2016;68:1-26.

7. Lu PJ, O'Halloran A, Williams WW, Lindley MC, Farrall S, Bridges $\mathrm{CB}$. Racial and ethnic disparities in vaccination coverage among adult populations in the U.S. Vaccine 2015;33 Suppl 4:D83-91.

8. van Dartel SA, Fransen J, Kievit W, Dutmer EA, Brus HL, Houtman $\mathrm{NM}$, et al. Predictors for the 5-year risk of serious infections in patients with rheumatoid arthritis treated with anti-tumour necrosis factor therapy: a cohort study in the Dutch Rheumatoid Arthritis Monitoring (DREAM) registry. Rheumatology 2013;52:1052-7.

9. Lau D, Hu J, Majumdar SR, Storie DA, Rees SE, Johnson JA. Interventions to improve influenza and pneumococcal vaccination rates among community-dwelling adults: a systematic review and meta-analysis. Ann Fam Med 2012;10:538-46.

10. Baker DW, Brown T, Lee JY, Ozanich A, Liss DT, Sandler DS, et al. A multifaceted intervention to improve influenza, pneumococcal, and herpes zoster vaccination among patients with rheumatoid arthritis. J Rheumatol 2016;43:1030-7.

11. Ledwich LJ, Harrington TM, Ayoub WT, Sartorius JA, Newman ED. Improved influenza and pneumococcal vaccination in rheumatology patients taking immunosuppressants using an electronic health record best practice alert. Arthritis Rheum 2009;61:1505-10.
12. Fiks AG, Hunter KF, Localio AR, Grundmeier RW, Bryant-Stephens T, Luberti AA, et al. Impact of electronic health record-based alerts on influenza vaccination for children with asthma. Pediatrics 2009;124:159-69.

13. Tuite AR, Fisman DN, Kwong JC, Greer AL. Optimal pandemic influenza vaccine allocation strategies for the Canadian population. PLoS One 2010;5:e10520.

14. ACIP Adult Immunization Work Group, Bridges CB, Woods L, Coyne-Beasley T; Centers for Disease Control and Prevention (CDC). Advisory Committee on Immunization Practices (ACIP) recommended immunization schedule for adults aged 19 years and older-United States, 2013. MMWR Suppl 2013;62:9-19.

15. Wolfe F, Kleinheksel SM, Cathey MA, Hawley DJ, Spitz PW, Fries JF. The clinical value of the Stanford Health Assessment Questionnaire Functional Disability Index in patients with rheumatoid arthritis. J Rheumatol 1988;15:1480-8.

16. Siriwardena AN. Targeting pneumococcal vaccination to high-risk groups: a feasibility study in one general practice. Postgrad Med J 1999;75:208-12.

17. Sittig DF, Teich JM, Osheroff JA, Singh H. Improving clinical quality indicators through electronic health records: it takes more than just a reminder. Pediatrics 2009;124:375-7.

18. Ng B, McBain L, Grainger R. Rheumatologists fail to advise people with RA to get immunised, which matters if you are under 65: An audit in a New Zealand rheumatology service. N Z Med J 2016;129:72-8.

19. Sandler DS, Ruderman EM, Brown T, Lee JY, Mixon A, Liss DT, et al. Understanding vaccination rates and attitudes among patients with rheumatoid arthritis. Am J Manag Care 2016;22:161-7. 